\title{
Compressed Sensing Based Fingerprint Identification for Wireless Transmitters
}

\author{
Caidan Zhao, ${ }^{1}$ Xiongpeng Wu, ${ }^{1}$ Lianfen Huang, ${ }^{1}$ Yan Yao, ${ }^{1}$ and Yao-Chung Chang ${ }^{2}$ \\ ${ }^{1}$ Department of Communication Engineering, Xiamen University, Xiamen, Fujian 361005, China \\ ${ }^{2}$ Department of Computer Science and Information Engineering, National Taitung University, Taitung 95092, Taiwan
}

Correspondence should be addressed to Yao-Chung Chang; ycc.nttu@gmail.com

Received 12 March 2014; Accepted 1 April 2014; Published 29 April 2014

Academic Editor: Han-Chieh Chao

Copyright (C) 2014 Caidan Zhao et al. This is an open access article distributed under the Creative Commons Attribution License, which permits unrestricted use, distribution, and reproduction in any medium, provided the original work is properly cited.

\begin{abstract}
Most of the existing fingerprint identification techniques are unable to distinguish different wireless transmitters, whose emitted signals are highly attenuated, long-distance propagating, and of strong similarity to their transient waveforms. Therefore, this paper proposes a new method to identify different wireless transmitters based on compressed sensing. A data acquisition system is designed to capture the wireless transmitter signals. Complex analytical wavelet transform is used to obtain the envelope of the transient signal, and the corresponding features are extracted by using the compressed sensing theory. Feature selection utilizing minimum redundancy maximum relevance ( $\mathrm{mRMR}$ ) is employed to obtain the optimal feature subsets for identification. The results show that the proposed method is more efficient for the identification of wireless transmitters with similar transient waveforms.
\end{abstract}

\section{Introduction}

Fingerprint identification for wireless transmitters [1-3] is of great significance in safe radio communications [47], military communications confrontation, civilian radio monitoring, and so forth [8-11]. Owing to the discreteness of the wireless transmitter devices and inconsistent manufacturing levels $[12,13]$, the inherent fingerprint features can be extracted from the emitted signals. Hence, different transmitters can be identified from the captured signals in a fast way $[14,15]$.

The key process of individual identification is to extract the unique signal features that form a valid device fingerprint. The fingerprint of a transmitter should distinctly characterize it from the rest of the transmitters through its unique features presented in the signal waveform. The transient behavior varies from different transmitters due to imperfections in the analog components. Additionally, some wireless transmitter signals, such as the WiFi signal, are sent by frame under the conflict avoidance protocol, which facilitates transient signal acquisition. Therefore, the unique features extracted from transient signals can make a distinction among different transmitters by adopting the intercepted communication signals [16].
The transient feature extraction mostly utilizes the wavelet analysis or envelope analysis in the time-domain. Choe et al. have used the orthogonal Daubechies- 4 wavelet to extract the transient signal features which are applied to identify two types of wireless transmitters at a recognition rate of $94.3 \%$ [17]. In the literature [18], wavelet coefficients are selected by genetic algorithms, and the rate of identification for communication stations is more than $90 \%$. Statistical features are extracted from the energy envelopes of seven different manufacturers' Bluetooth devices by Rehman et al., and the average recognition rate reaches up to $99.9 \%$ [19]. In the literature [20], the energy envelope, obtained from the instantaneous signal by spectrogram analysis, is polynomial fitted to get the fitting coefficient characteristics. The result illustrates that these coefficient characteristics can effectively distinguish four different network cards. Zhao et al. have utilized complex analytic wavelet transform to obtain the envelopes from the signals and extracted the fitting coefficient characteristics using the Gaussian fitting method. The identification rate is up to $93 \%$ [21]. However, the characteristics of wavelet coefficients extracted by the wavelet analysis methods are of little difference and with large number. It requires high recognition performance of the recognizer. In addition, the above mentioned methods result in large differences of the 


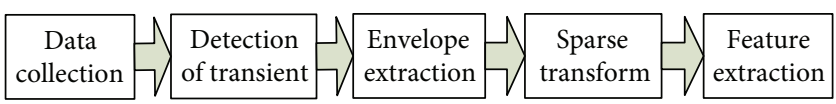

FIGURE 1: The flowchart of feature extraction for wireless transmitters.

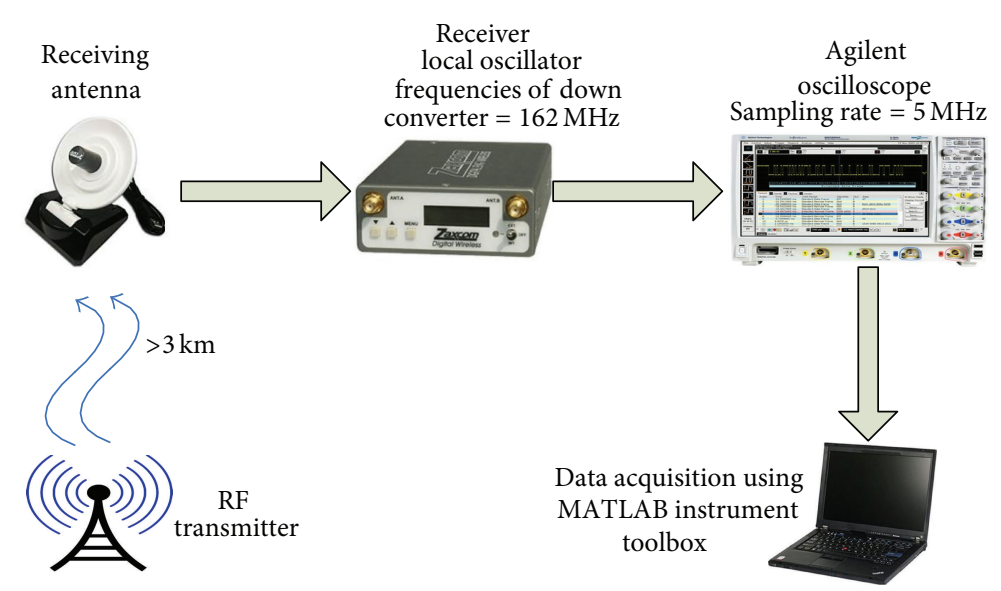

FIGURE 2: The block diagram of the signal acquisition for wireless transmitters.

signal waveforms, and most of their applications are for the short-distance WiFi signals and Bluetooth signals.

The compressed sensing theory can make the dimension of signals lower and all the information of original signal cannot be lost [22], and, therefore, this paper proposes a new method to identify different wireless transmitters based on compressed sensing to deal with the identification signals of high attenuation, long-distance transmission, and high transient waveform similarity. Experiments have been carried out in an outdoor environment. The distance between the transceivers is more than $3 \mathrm{~km}$. Complex analytical wavelet transform is used to obtain the envelope of the transient signal, and features are extracted from the envelope based on the compressed sensing theory. A feature selection utilizing minimum redundancy maximum relevance (mRMR) is adopted to obtain optimal feature subsets for identification. The proposed method has also been compared to the techniques reported in the literatures.

The rest of this paper is organized as follows. In Section 2, signals acquisition is introduced. Furthermore, transient, envelope, and feature extraction of wireless transmitters is explained. Section 3 gives an overview of analysis of recognition results and draws a comparison between the proposed technique and the reported techniques. Finally, the conclusion follows.

\section{Methodology}

The key step of fingerprint identification for the wireless transmitter is to extract unique features from the signals that different transmitters emit. To use compressed sensing theory, the sparseness of the signal has to be satisfied. However, lots of high frequency coefficients under the sparsedomain exist in a high-frequency signal, such as the wireless signal. Besides, sparse transform from a transmitter signal envelope can greatly increase the sparseness and it is convenient to extract the features using the compressed sensing. The proposed technique of features extraction is shown in Figure 1. Firstly, the envelope is extracted from the signals of starting-point detected according to the complex analytic wavelet transform. Then, the features are obtained from the envelope by utilizing the compressed sensing theory.

2.1. Data Collection. Figure 2 shows the signal acquisition diagram. The sampling frequency is set as $5 \mathrm{MHz}$. The distance between the transmitting antenna and the receiving antenna is over $3 \mathrm{~km}$. After being down-converted by the receiver, the intermediate-frequency signal is collected by an oscilloscope. The MATLAB instrument toolbox is used for transient and feature extraction. Due to the environmental impact, the receiving SNR of signals is around $10 \mathrm{~dB}$ to $20 \mathrm{~dB}$. The transient signal waveforms of 8 different wireless transmitters, which are used for recognition analysis, are actually acquired as shown in Figure 3. The figure shows that there is no substantial difference among these waveforms. A total amount of 200 waveforms were captured and stored from each wireless transmitter during the device discovery mode.

2.2. Transient Extraction. Accurate transient detection is the key step to extract the features. Huang et al. have proposed a method of mean change point detection [23] to analyze the transient based on phase detection [24]. Its idea is to magnify the difference between the statistic of samples before and after the section, and the position of the maximum difference is determined to be the start of transient. The principle is as follows.

The sample sequence $x_{1}, x_{2}, \ldots, x_{N}$ is known $\left(x_{i}\right.$ corresponds to the phase variance in the method of phase detection). 

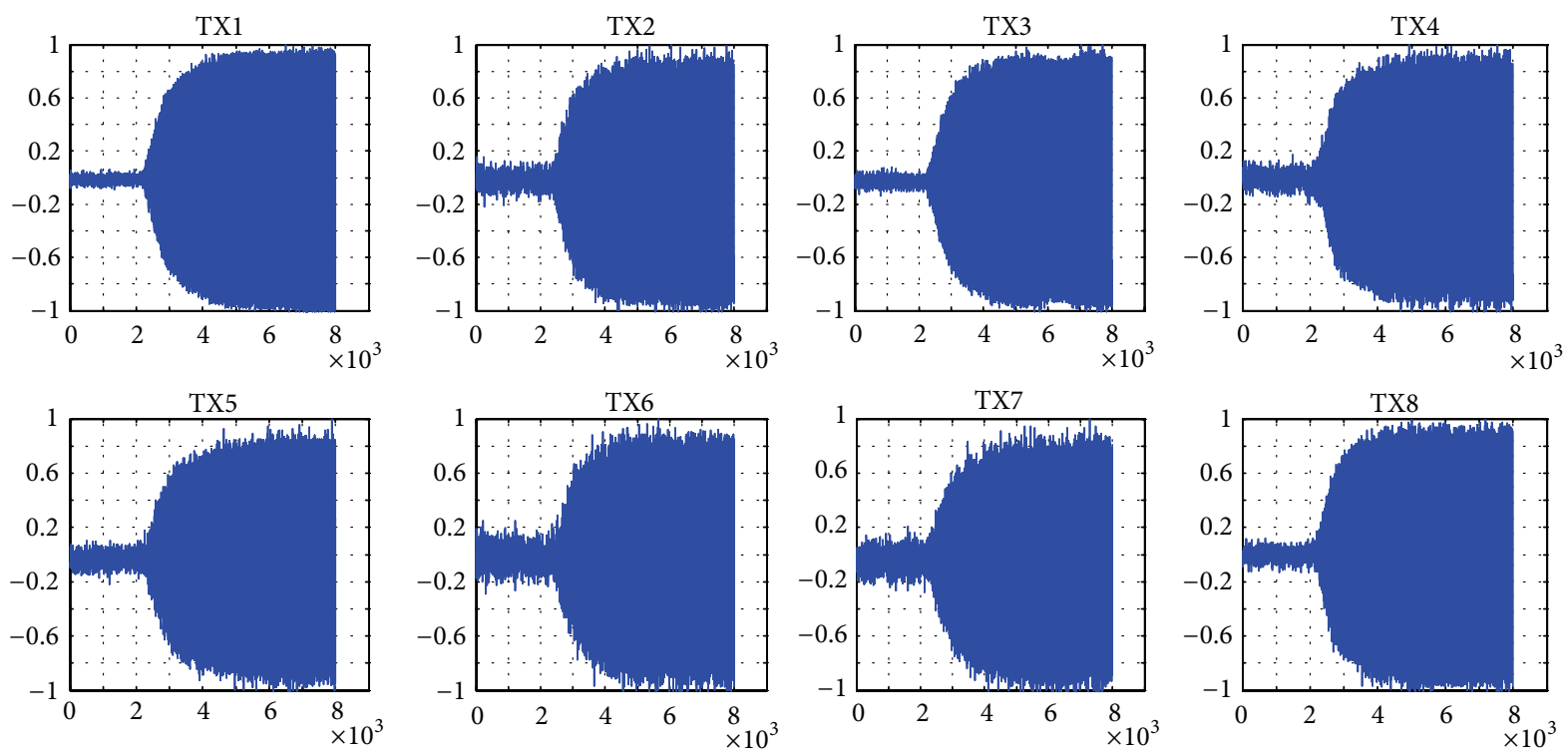

FIGURE 3: The transient waveforms of 8 different wireless transmitters.

Firstly, let $i=2,3, \ldots N$. The samples can be divided into two sections: $x_{1}, x_{2}, \ldots, x_{i-1}$ and $x_{i}, x_{i+1}, \ldots, x_{N}$ for each number of $i$; then we can calculate the mean and statistics of each section. Consider

$$
S_{i}=\sum_{t=1}^{i-1}\left(x_{t}-\overline{X_{i 1}}\right)^{2}+\sum_{t=i}^{N}\left(x_{t}-\overline{X_{i 2}}\right)^{2}
$$

Secondly, $\bar{X}$ and $S$ are calculated, which represent the average and statistics of the original sample, respectively. Consider

$$
S=\sum_{t=1}^{N}\left(x_{t}-\bar{X}\right)^{2}
$$

Thirdly, the curve of $S-S_{i}$ is plotted. The position of maximum of the curve would be the change point.

As shown in Figure 4, the detection for the start of wireless transmitter transient is accurate. Compared with the method of variance fractal dimension threshold detection [25], this method can increase the accuracy of detection without the limit of a threshold.

2.3. Envelope Extraction. In a wireless communication environment, because of the interference of the background noise, signal envelope extracted by conventional Hilbert transform, which is shown in Figure 5(b), cannot effectively eliminate the effect of the random noise. It causes an adverse influence on the subsequent feature extraction [26]. In the literature $[19,20]$, the authors have proposed a method of spectrogram analysis to obtain transient envelope. However, due to the limitation in short time window, the transient envelope exhibits an obvious distortion during the extraction process. This extraction result is shown in Figure 5(c).

Recently, many scholars have put forward the method to extract the envelope from the signal based on complex

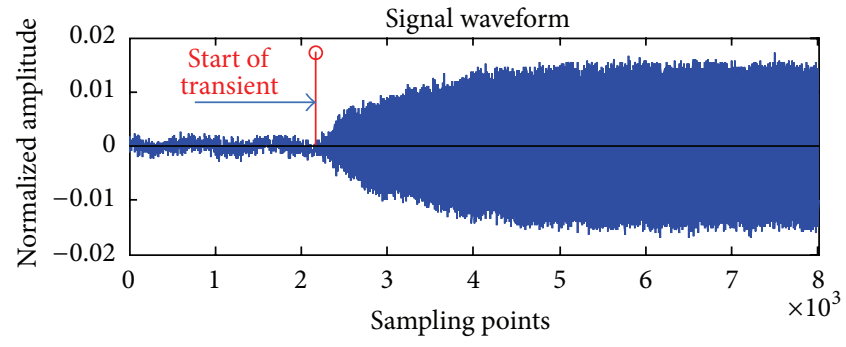

(a)

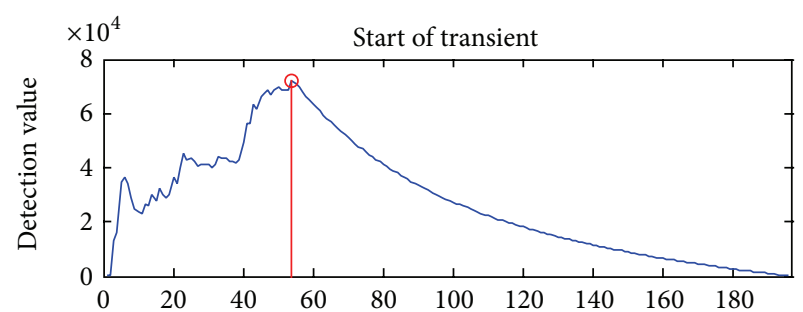

(b)

FIGURE 4: The detection for the start of wireless transmitter transient.

analytical wavelet transform [26-28]. The envelope obtained by this method has an inhibited effect on random noise and shows a smooth envelope curve whose distortion is relatively petty.

The simplified expression of Morlet wavelet is as follows:

$$
\phi(t)=\frac{1}{\sqrt{2 \pi \sigma^{2}}} e^{-i 2 \pi f_{0} t} e^{-t^{2} / 2 \sigma^{2}}, \quad \sigma>0,
$$

where $\sigma$ is the wavelet shape parameter (also known as bandwidth) and $f_{0}$ is the wavelet center frequency. 


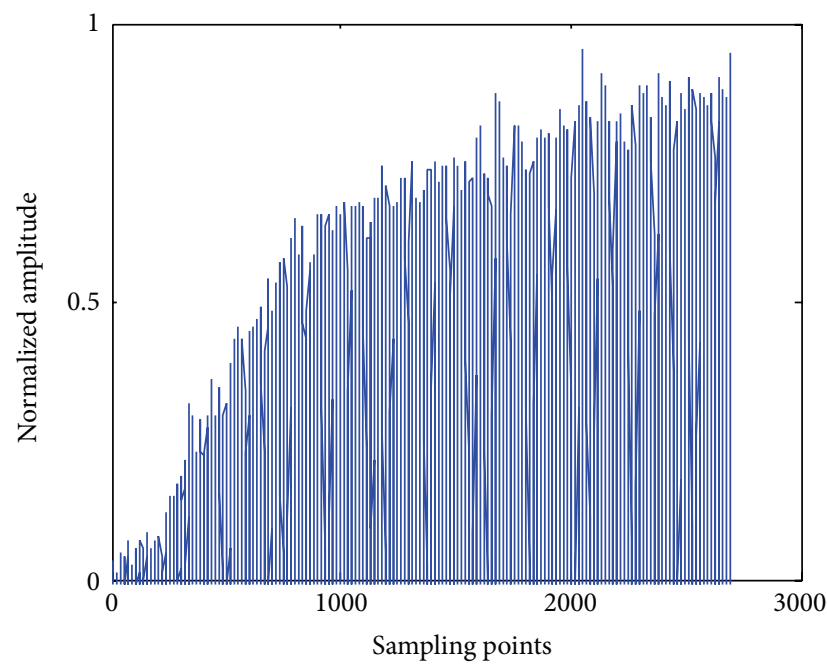

(a)

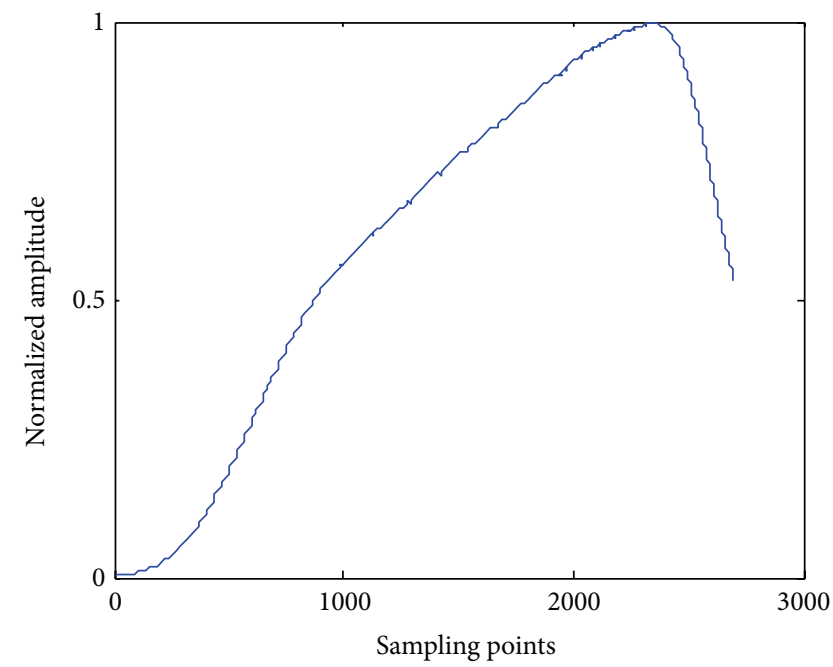

(c)

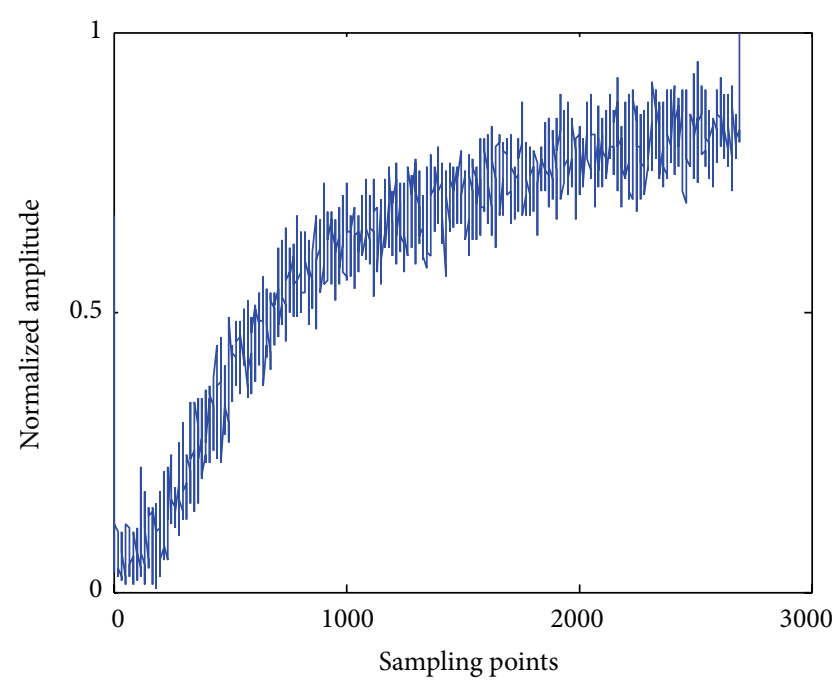

(b)

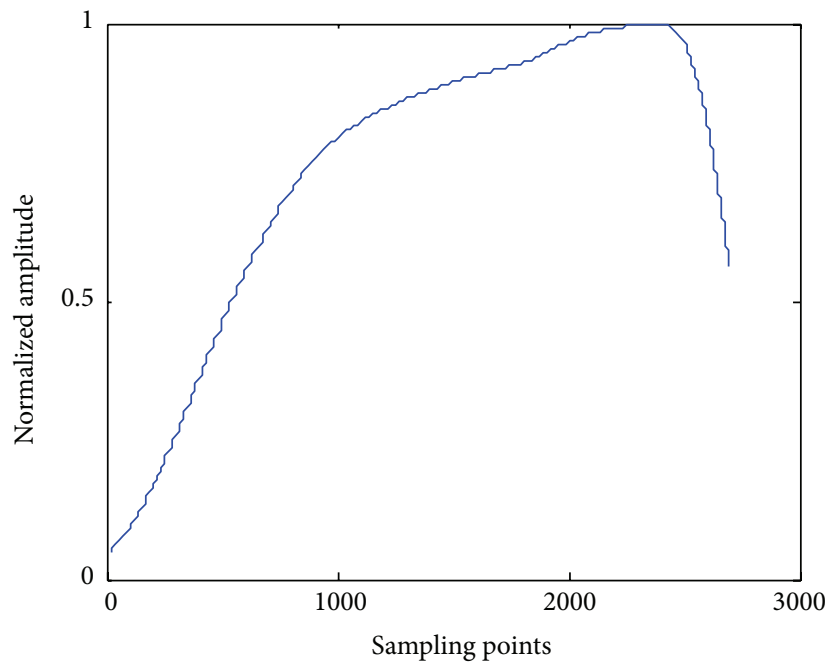

(d)

FIGURE 5: A comparison between Hilbert transform, spectrum slice analysis, and wavelet transform.

For time-discrete signa $x(t), t=n \Delta T, n=1,2, \ldots, N-1$, where $\Delta T$ is the sampling period, Morlet wavelet transform of the signal can be expressed as follows:

$$
\begin{aligned}
W_{x}(b, a)(m)= & \frac{\Delta T}{a \sqrt{2 \pi \sigma^{2}}} \sum_{n=0}^{N-1} x(n) \exp \left[-\frac{(n \Delta T-b)^{2}}{2 a^{2} \sigma^{2}}\right] \\
& \times \exp \left(-i 2 \pi f_{0} \frac{n \Delta T-b}{a}\right),
\end{aligned}
$$

where $b=m \Delta T, m=1,2, \ldots, N-1, a$ is a scale factor. When $a=f_{0} / f_{1}, W_{x}(b, a)(m)$ can get its maximum value. In the expression of $a=f_{0} / f_{1}, f_{0}$ is the wavelet center frequency and $f_{1}$ is the signal frequency. In order to ensure that the signal amplitude is equal before and after transformation, the envelope amplitude of the signal is $2\left|\mathrm{~W}_{x}(b, a)(m)\right|$.

To obtain a smooth envelope curve, the parameter $\sigma$ and $f_{0}$ of Morlet mother wavelet is adjusted. After lots of experiments, as shown in Figure 5(d), when $\sigma=7$ and $f_{0}=$ 1 , the envelope curve is similar to the original signal and smoother.

The envelope extraction of 8 different wireless transmitters using complex analytical wavelet transform is shown in Figure 6. It can be seen that the envelopes have some differences between each other.

2.4. Feature Extraction Based on Compressed Sensing. The goal of the feature extraction is to form a unique transmitter fingerprint, which distinctly characterizes a transmitter from all of the transmitters. The method of this paper is to extract a feature from the envelope based on compressed sensing.

An orthogonal (sparse) transformation such as DCT (discrete cosine transform) [29] and DWT (discrete wavelet transform) [30] is performed for the envelope signal. 


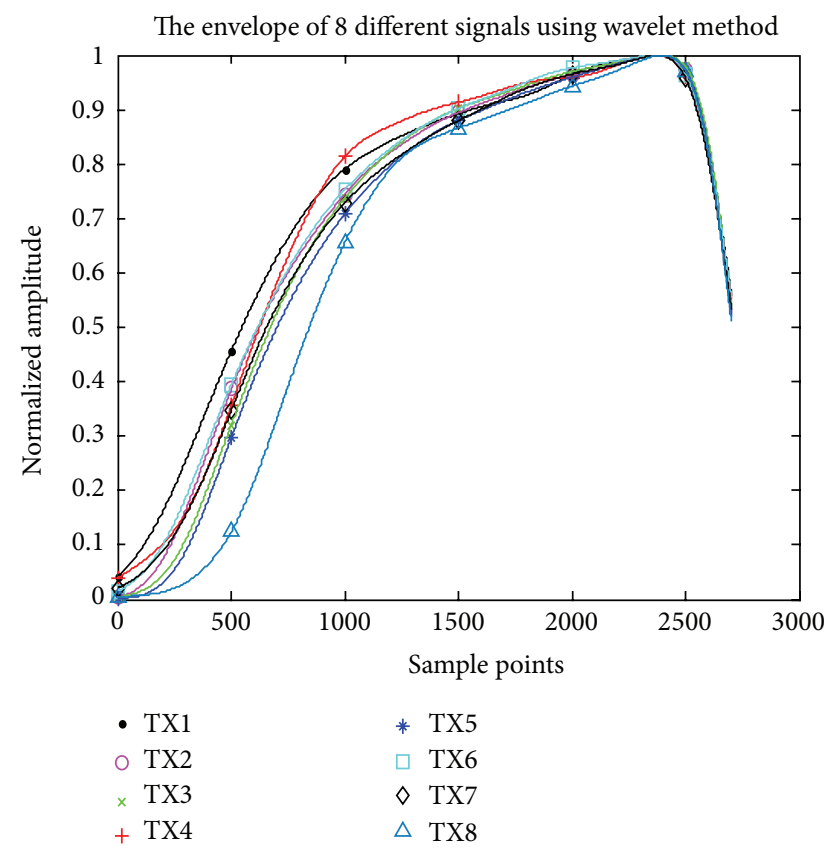

Figure 6: The envelope extraction of 8 different wireless transmitters using complex analytical wavelet transform.

Assuming the length of the envelope signal $y$ is $N, y$ is able to be represented by the linear combination of $\psi=$ $\left\{\psi_{1}, \psi_{2} \cdots \psi_{N}\right\}$. Consider

$$
y=\sum_{n=1}^{N} \psi_{n} s_{n}=\psi s,
$$

where $\psi$ is an $N \times N$ orthogonal matrix to form an orthogonal transformation and $s$ is an $N \times 1$ coefficient vector. When $s$ has $K(K \ll N)$ nonzero coefficients, the signal $y$ is sparse under the matrix $\psi$.

(1) Linear measurement is the key process of the compressed sensing theory. The signal $y$ is mapped to a measurement vector $z$ which is a lower dimensional data. Consider

$$
z=\Phi y=\Phi \psi s,
$$

where $z$ is an $M \times 1(M<N)$ vector, namely, the extracted features and $\Phi$ is an $M \times N$ measurement matrix. There are four kinds of measurement matrices including Gauss matrix $[22,31]$, Bernoulli matrix [22, 31], Toeplitz matrix [32], and sparse matrix [33]. The measurement matrix is critical for feature extraction. It is required that the measurement matrix can make the dimension of feature vector $z$ as low as possible and all information of original signal cannot be lost.

(2) The reconstruction algorithm is used to confirm whether the features contain all information of the original signal, that is to say, whether the measurement $z$ can recover the original signal $y$. The most direct way to reconstruct the signal is optimization problems in $L 0$ norm:

$$
\begin{array}{ll}
\min & \|s\|_{l 0} \\
\text { s.t. } & z=\Phi \psi s,
\end{array}
$$

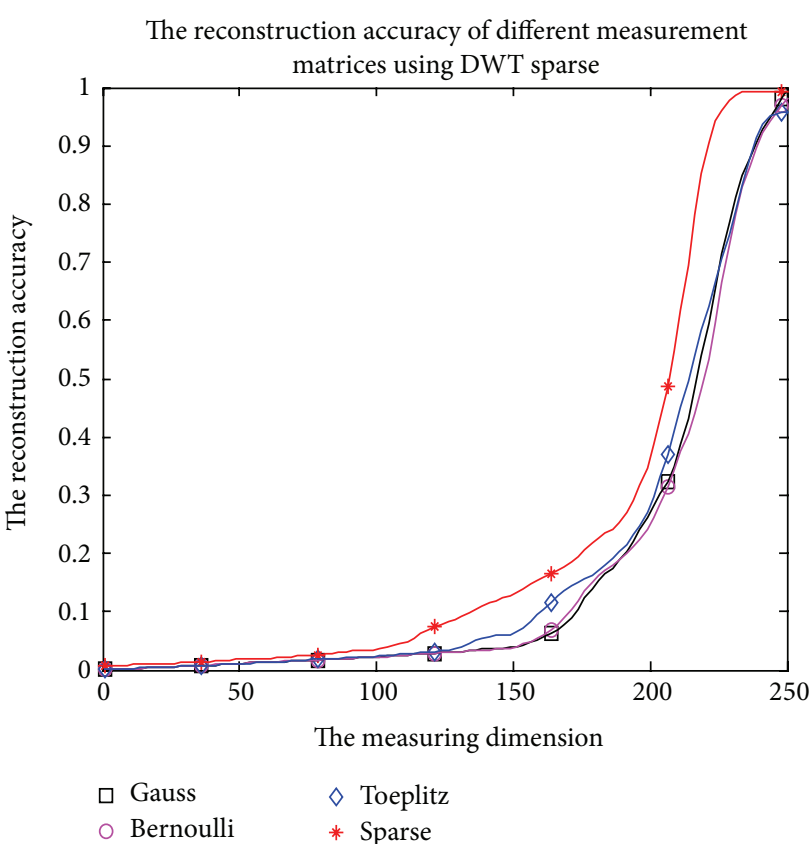

(a)

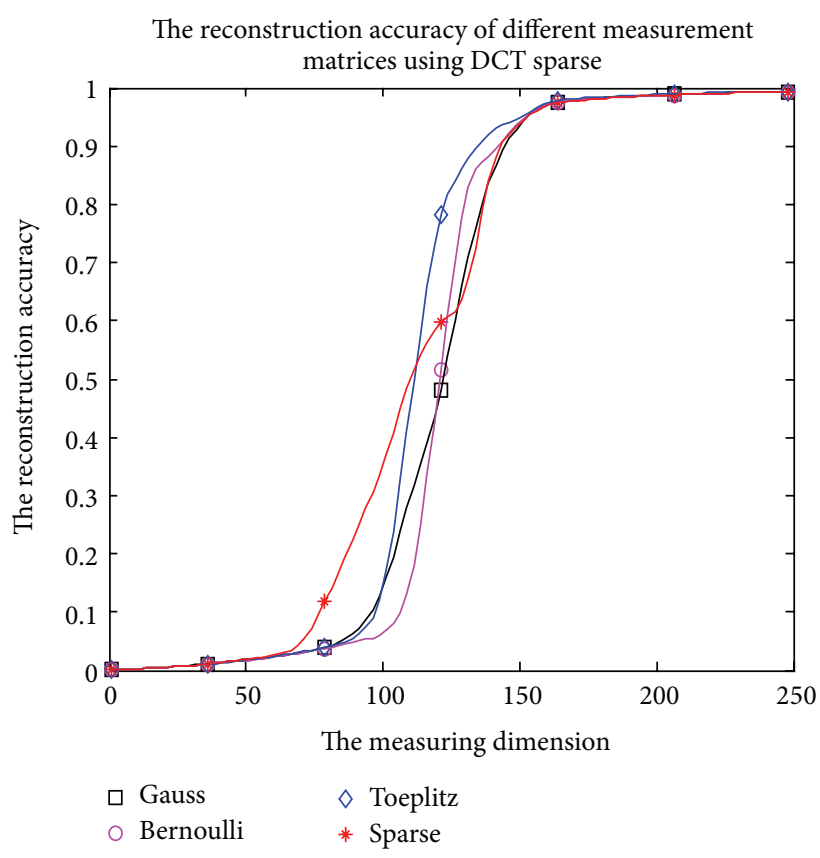

(b)

FIGURE 7: The reconstruction accuracy change with the measurement matrices and the dimension $M$ of measurement vector $z$.

where $s$ is the sparse coefficients vector for reconstruction. Since the optimal computational complexity for reconstructing the signal is very high, Bayesian algorithm (BCS) [30] is usually used to find the approximate solution under acceptable complexity. Finally, the reconstruction signal $\tilde{y}$ is solved by Formula [16].

It is depicted in Figure 7 that the reconstruction accuracy changes with the measurement matrices and the dimension 


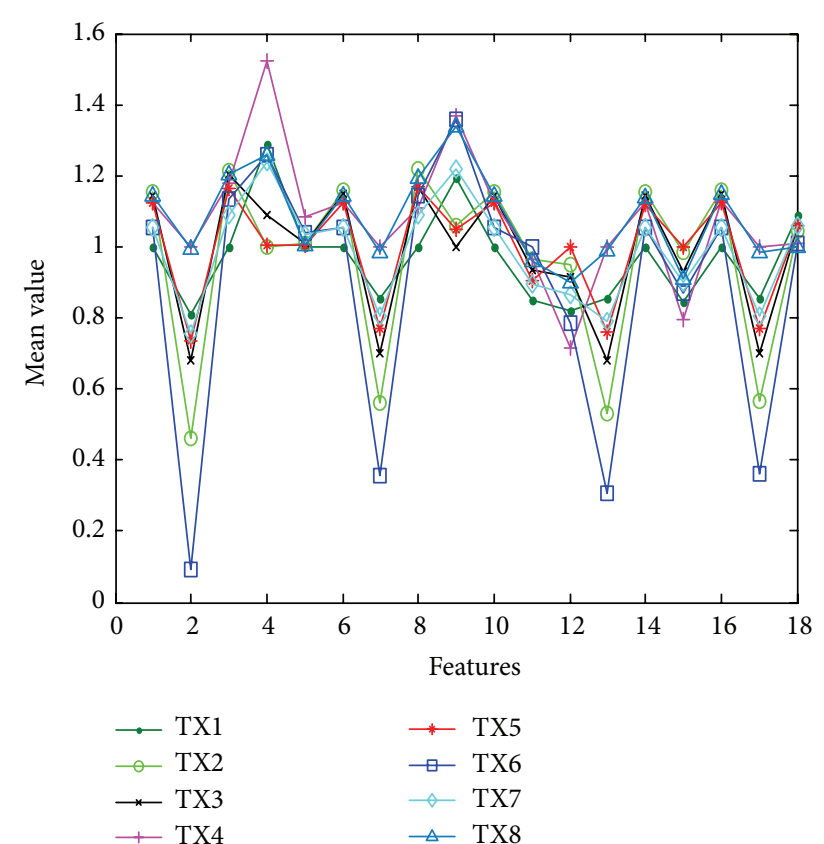

FIGURE 8: Averages of 18 features extracted from 100 groups of signals for 8 wireless transmitters.

$M$ of measurement vector $z$. It can be clearly shown that the sparse matrix is best suited for feature extraction. Taking into account the reconstruction accuracy, the dimension of the feature vector is set to 230 for DWT, while the dimension of feature vector is set to 160 for DCT.

(3) From the previous analysis, two feature vectors are extracted based on the DCT and DWT.

\section{Recognition Results Analysis}

In the stage of signal analysis, a recognizer combining multiclassification SVM recognizer [34] with BP neural network recognizer [35] is established. The "one-against-all" method needs more training time. And the appropriate parameters of multiclassification SVM are obtained by cross validation on the training data. To realize the multiclassification SVM, it is feasible to choose the "one-against-one" method, select the Gaussian radial basis function as Kernel, and set nuclear parameter and penalty factor as 1 and 1000, respectively. Furthermore, BP neural network recognizer is built by a three-layer neural network which contains only one hidden layer. The input layer neurons are set to be the number of dimensions of the transient fingerprint feature vector, and the output layer neurons are set to be the number of stations that need to be classified.

As presented in this paper, the number of features extracted by the compressed sensing is too much, which results in a long time identification. The recognition rate decreases for some irrelevant feature interferences. Thus, the use of mRMR [36] to optimize the features is considerable. To directly analyze the effectiveness of the features we select, the distribution of 18 features' average values extracted from
TABLE 1: Classification results for different wireless transmitters.

\begin{tabular}{cccccccccc}
\hline \multirow{2}{*}{$\begin{array}{c}\text { Input } \\
\end{array}$} & TX1 & TX2 & TX3 & TX4 & TX5 & TX6 & TX7 & TX8 & rate (\%) \\
\hline TX1 & $\mathbf{9 0}$ & 0 & 2 & 0 & 0 & 3 & 5 & 0 & 90 \\
TX2 & 1 & $\mathbf{8 0}$ & 6 & 1 & 5 & 7 & 0 & 0 & 80 \\
TX3 & 1 & 9 & $\mathbf{8 8}$ & 1 & 0 & 1 & 0 & 0 & 88 \\
TX4 & 3 & 0 & 0 & $\mathbf{9 1}$ & 0 & 2 & 0 & 4 & 91 \\
TX5 & 0 & 5 & 1 & 0 & $\mathbf{9 3}$ & 0 & 0 & 1 & 93 \\
TX6 & 3 & 2 & 2 & 5 & 3 & $\mathbf{7 9}$ & 3 & 3 & 79 \\
TX7 & 5 & 0 & 2 & 3 & 0 & 2 & $\mathbf{8 8}$ & 0 & 88 \\
TX8 & 1 & 0 & 1 & 2 & 1 & 0 & 2 & $\mathbf{9 3}$ & 93 \\
\hline
\end{tabular}

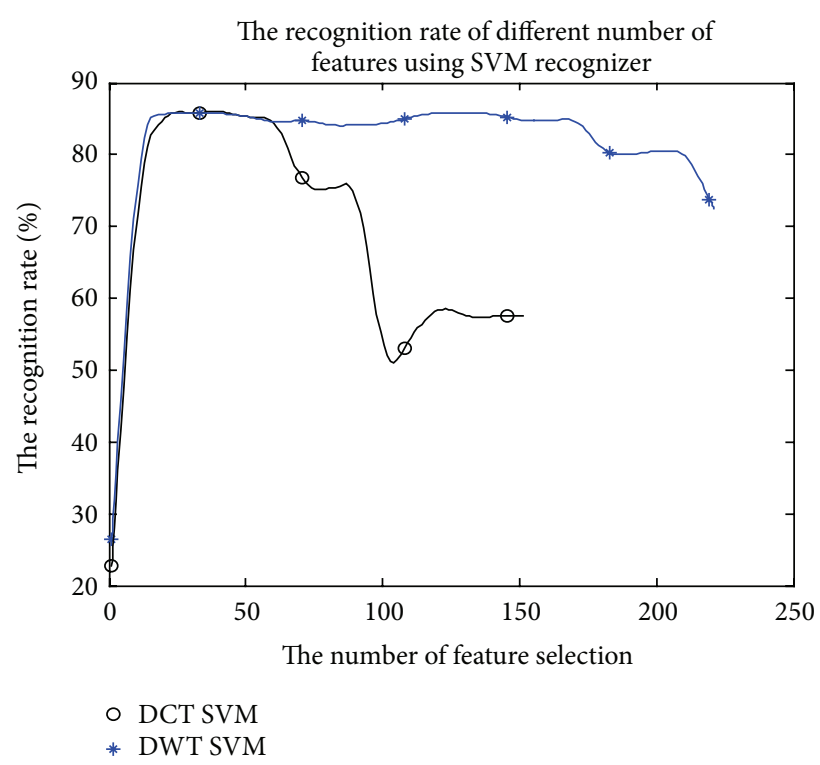

FIGURE 9: The comparison of the recognition results based on different sparse bases.

the 100 groups of signal of 8 wireless transmitters is shown in the Figure 8. It can be seen from the picture that the mRMR algorithm is an effective way to distinguish different wireless transmitters.

Figure 9 shows that the SVM identification results, which are through the envelope signal sparse by the DCT and DWT, with mRMR method to select features. The identification effectiveness is changing with the number of selecting features and different properties of sparse bases. In general, the convergence of DCT sparse base is better. If the number of the selecting features is under 50 , the identification rate can be above $80 \%$.

Through the envelope signal sparse by DCT sparse base, the SVM recognizer is utilized to identify 8 different wireless transmitters. For each transmitter, 100 groups are used for training and 100 groups for testing. Table 1 presents the specific identification results. The data illustrate that the algorithm referred to in this paper is effective to identify transmitter when the average identification rates of most transmitters are over $88 \%$ and the average identification rate is $87.75 \%$. 
TABLE 2: Comparison of identification of a transmitter using three different methods.

\begin{tabular}{lccc}
\hline Comparison metric & Mathematical statistics method & Fitting method & $\begin{array}{c}\text { Compressed sensing } \\
\text { method }\end{array}$ \\
\hline RF fingerprints & $\begin{array}{c}\text { Area under the normalized curve, } \\
\text { duration, maximum slope, kurtosis, } \\
\text { skewness, and variance of curve }\end{array}$ & Gaussian fitting coefficients & $\begin{array}{c}\text { Feature extracted as } \\
\text { explained in subsection II }\end{array}$ \\
Envelope extraction & STFT slice method & $\begin{array}{l}\text { Wavelet method } \\
\text { STFT slice method }\end{array}$ & Wavelet method \\
Classifier & KNN & SVM and BP & SVM and BP \\
\hline
\end{tabular}

TABLE 3: Performance comparison for the envelope extraction and feature extraction.

\begin{tabular}{|c|c|c|c|c|c|}
\hline & \multicolumn{2}{|c|}{ Envelope extraction } & \multicolumn{3}{|c|}{ Feature extraction } \\
\hline & STFT slice & Wavelet & $\begin{array}{l}\text { Mathematical } \\
\text { statistics }\end{array}$ & Fitting & Compressed sensing \\
\hline Advantage & $\begin{array}{l}\text { Eliminate the effect } \\
\text { of the noise }\end{array}$ & $\begin{array}{l}\text { Envelope is smoother } \\
\text { and precise }\end{array}$ & $\begin{array}{l}\text { Simple; the feature } \\
\text { is easy to extract }\end{array}$ & $\begin{array}{l}\text { Simple; the features } \\
\text { are easy to extract }\end{array}$ & $\begin{array}{c}\text { The features are of high } \\
\text { representativeness }\end{array}$ \\
\hline Disadvantage & $\begin{array}{l}\text { Envelope exhibits a } \\
\text { distortion }\end{array}$ & $\begin{array}{l}\text { Parameters are } \\
\text { determined by } \\
\text { experience }\end{array}$ & $\begin{array}{l}\text { Some features are } \\
\text { not representative }\end{array}$ & $\begin{array}{l}\text { Fitting function is } \\
\text { determined by } \\
\text { experience }\end{array}$ & $\begin{array}{l}\text { Complex; need a long } \\
\text { time }\end{array}$ \\
\hline Complexity & $O\left(n^{2}\right)$ & $O\left(n^{2}\right)$ & $O(n)$ & $O(n)$ & $O\left(n^{2}\right)$ \\
\hline Time for calculation (s) & 2.251245 & 3.568356 & 1.324417 & 2.419341 & 4.626052 \\
\hline
\end{tabular}

The method proposed in this paper is compared with the works of Rehman et al. [19] and Zhao et al. [20, 21]. Rehman's work simply extracts the envelope statistic features from the energy spectrum envelope. And Zhao et al. put forward using the fitting coefficients of the envelope as the signal features for identification. Table 2 describes the comparison among Reham's work, Zhao's work, and the proposed work in this paper. The term mathematical statistics method refers to Rehman et al.'s work, fitting method is used for Zhao et al's work, and compressed sensing method represents the work proposed in this paper. The $8 \mathrm{RF}$ signal transmitting devices identified in this paper are similar in their transient waveforms. The recognition rates of either Rehman's or Zhao's method are relatively lower. Figure 10 shows the specific comparison result. As shown in the figure, the extracted envelope features method based on compressive sensing theory in this paper has many advantages over the existing statistic features and fitting coefficient characteristics methods. On the one hand, extracting envelope by complex analytic wavelet is superior to spectrum slicing. On the other hand, the recognition performance of SVM recognizer is better than both BP neural network recognizer and $\mathrm{KNN}$ recognizer.

According to the disadvantages of the previous methods and the merits of our proposed algorithm, performance comparison for the envelope extraction and feature extraction is provided as Table 3.

\section{Conclusions}

This paper has proposed a fingerprint identification method for wireless transmitter signal based on compressed sensing. Complex analytical wavelet transform is used to obtain the envelope of the transient signal, and features are extracted

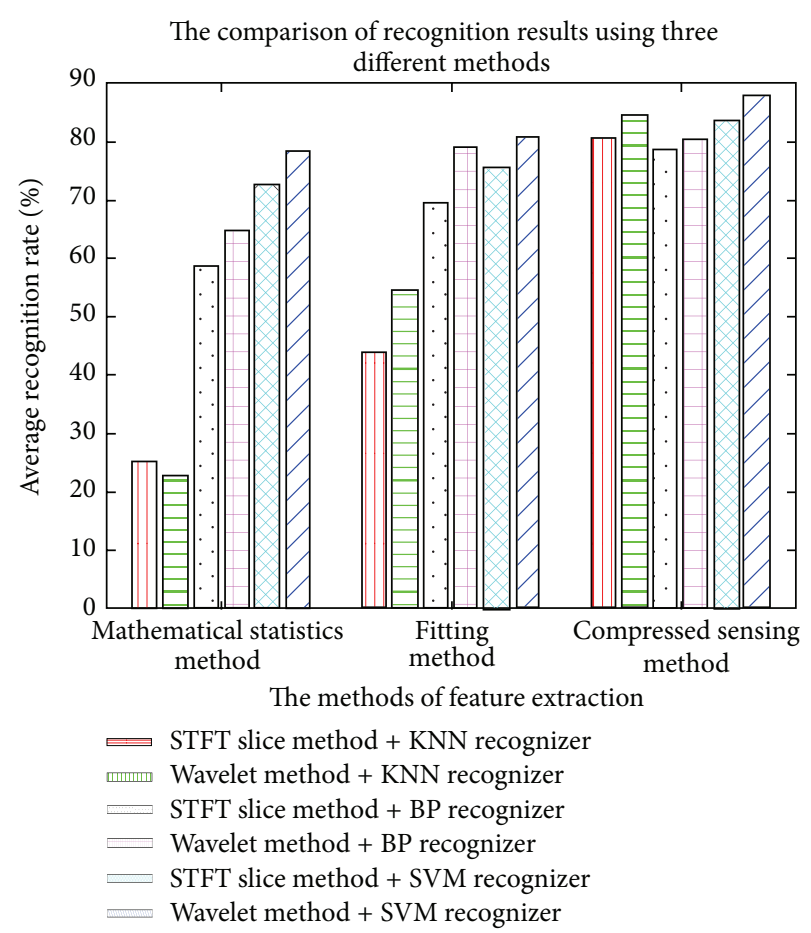

FIgURE 10: The comparison of classification results by three different methods.

from the envelope using the compressed sensing theory. A feature selection utilizing minimum redundancy maximum relevance (mRMR) is employed to obtain optimal feature subsets for identification. Finally, the recognition of 8 wireless transmitters by the SVM recognizer and BP neural network recognizer is completely performed. From a series of experiments, it can be concluded that the method proposed in 
the paper can effectively identify the transmitter signals, whose transient waveforms are difficult to distinguish with the naked eye. Especially, the method put forward in the paper has better performance in the recognition of wireless transmitter signals with serious interference and signal attenuation when compared to the previous methods, such as extracting statistic features directly from the envelope, fitting coefficient characteristic. What is more, this method may be applied to identify the signals which are transmitted by the transmitters with the same batch and type. According to the results, a future research on the optimization of the Morlet wavelet's parameter is expected to improve the performance of the proposed method.

\section{Conflict of Interests}

The authors declare that there is no conflict of interests regarding the publication of this paper.

\section{Acknowledgments}

This work is partly supported by NSFC (61271242, 61001072), NCETFJ, and the Fundamental Research Funds for the Central Universities (2012121028).

\section{References}

[1] S.-C. Kwon, Y.-T. Lee, and J.-S. Seo, "A novel transmitter identification technique for use in distributed 8 VSB DTV system," in Proceedings of the 63rd IEEE Vehicular Technology Conference (VTC '06), pp. 2211-2215, July 2006.

[2] H. H. Cho, C. Y. Chen, T. K. Shih, and H. C. Chao, "A survey on underwater delay/disruption tolerant wireless sensor network routing," to be published in IET Wireless Sensor Systems.

[3] H.-C. Chu, D.-J. Deng, and H.-C. Chao, "The digital forensics of portable electronic communication devices based on a Skype im session of a pocket PC for NGC," Wireless Communications and Mobile Computing, vol. 11, no. 2, pp. 211-225, 2011.

[4] C.-Y. Chen, K.-D. Chang, and H.-C. Chao, "Transactionpattern-based anomaly detection algorithm for IP multimedia subsystem," IEEE Transactions on Information Forensics and Security, vol. 6, no. 1, pp. 152-161, 2011.

[5] Y. C. Chang, "Heterogeneous wireless sensor network with EPC network architecture for U-life environment," to be published in Journal of Internet Technology.

[6] Y. M. Huang, M. Y. Hsieh, H. C. Chao, S. H. Hung, and J. H. Park, "Pervasive, secure access to a hierarchical sensor-based healthcare monitoring architecture in wireless heterogeneous networks," IEEE Journal on Selected Areas in Communications, vol. 27, no. 4, pp. 400-411, 2009.

[7] Y. C. Chang and H. W. Wang, "Mobile business via cross layer approach toward intelligent RFID purchasing system," Journal of Internet Technology, vol. 11, no. 7, pp. 965-973, 2010.

[8] C.-S. Li, Y.-C. Tseng, H.-C. Chao, and Y.-M. Huang, "A neighbor caching mechanism for handoff in IEEE 802.11 wireless networks," Journal of Supercomputing, vol. 45, no. 1, pp. 1-14, 2008.

[9] N.-N. Lu, H.-K. Zhang, Y.-C. Chang, and H.-C. Chao, "IPas++: a novel accountable and scalable Internet Protocol for future internet," Journal of Internet Technology, vol. 12, no. 5, pp. 769780, 2011.
[10] L. Shu, M. Hauswirth, H.-C. Chao, M. Chen, and Y. Zhang, "NetTopo: a framework of simulation and visualization for wireless sensor networks," Ad Hoc Networks, vol. 9, no. 5, pp. 799-820, 2011.

[11] H.-C. Chu and W. D. Lin, "Live information discovery of the P2P instant messaging based on Skype's finger printing," Journal of Internet Technology, vol. 11, no. 2, pp. 193-202, 2010.

[12] Z.-W. Cai and J.-D. Li, "Study of transmitter individual identification based on bispectra," Journal on Communications, vol. 28, no. 2, pp. 75-79, 2007.

[13] D. Xu, H. Xu, Q. Lu et al., "A specific emitter identification method based on self-excitation oscillator mode," Signal Processing, vol. 24, no. 1, pp. 122-126, 2008.

[14] L. Xiao, W. S. Lin, Y. Chen, and K. J. R. Liu, "Indirect reciprocity security game for large-scale mobile wireless networks," IEEE Transactions on Information Forensics \& Security, vol. 7, no. 4, pp. 1368-1380, 2012.

[15] L. Xiao, H. Dai, and P. Ning, "MAC design of uncoordinated FH-based collaborative broadcast," IEEE Wireless Communications Letters, vol. 1, no. 3, pp. 261-264, 2012.

[16] Z. Shi, M. Liu, and L. Huang, "Transient-based identification of 802.11 b wireless device," in Proceedings of the International Conference on Wireless Communications and Signal Processing (WCSP '11), November 2011.

[17] H. C. Choe, C. E. Poole, A. M. Yu, and H. H. Szu, "Novel identification of intercepted signals from unknown radio transmitters," in Wavelet Applications II, vol. 2491 of Proceedings of SPIE, pp. 504-517, April 1995.

[18] M.-J. Lu, Y. Zhan, X.-C. Si, and X.-N. Yang, "Extraction of the transient characteristics of the communication radiating source and individual identification," Journal of Xidian University, vol. 36, no. 4, pp. 736-740, 2009.

[19] S. Ur Rehman, K. Sowerby, and C. Coghill, "RF fingerprint extraction from the energy envelope of an instantaneous transient signal," in Proceedings of the Australian Communications Theory Workshop (AusCTW '12), pp. 90-95, February 2012.

[20] C. Zhao, L. Huang, L. Hu, and Y. Yao, "Transient fingerprint feature extraction for WLAN cards based on polynomial fitting," in Proceedings of the 6th International Conference on Computer Science and Education (ICCSE '11), pp. 1099-1102, August 2011.

[21] L. Huang, X. Wu, C. Zhao, and M. Gao, "Identification of radio transmitters fingerprint based on curve fitting," in Proceedings of the IEEE International Conference on Signal Processing, Communications and Computing (ICSPCC '13), 2013.

[22] D. L. Donoho, "Compressed sensing," IEEE Transactions on Information Theory, vol. 52, no. 4, pp. 1289-1306, 2006.

[23] L. Huang, M. Gao, C. Zhao, and X. Wu, "Detection of Wi-Fi transmitter transients using statistical method," in Proceedings of the IEEE International Conference on Signal Processing, Communications and Computing (ICSPCC '13), 2013.

[24] J. Hall, M. Barbeau, and E. Kranakis, "Detection of transient in radio frequency fingerprinting using signal phase," in Proceedings of the 3rd IASTED International Conference on Wireless and Optical Communications, pp. 13-18, July 2003.

[25] D. Shaw and W. Kinsner, "Multifractal modelling of radio transmitter transients for classification," in Proceedings of the IEEE WESCANEX Conference on Communications, Power and Computing Conference, pp. 306-312, May 1997.

[26] T. Hou, "Detection of signal envelope based on the complex analytical wavelet transform," Journal of Xian University of Posts and Telecommunication, vol. 16, no. 3, pp. 18-21, 2011. 
[27] C. Liu and Z. Qiu, "Extracting vibration signal envelopes based on Morlet wavelet transform," Journal of Circuits and Systems, vol. 5, no. 1, pp. 68-71, 2000.

[28] X. Yuan and J. Yu, "Complex analytical wavelet transform for the extraction and analysis of speech signal envelops," Acta Electronica Sinica, vol. 27, no. 5, pp. 142-144, 1999.

[29] I. Naseem, R. Togneri, and M. Bennamoun, "Sparse representation for speaker identification," in Proceedings of the 20th International Conference on Pattern Recognition (ICPR '10), pp. 4460-4463, August 2010.

[30] Y.-G. Cen and L.-H. Cen, "Sparse representation and reconstruction of signals based on the peak transform," Journal of Electronics \& Information Technology, vol. 33, no. 2, pp. 326-331, 2011.

[31] Y. Tsaig and D. L. Donoho, "Extensions of compressed sensing," Signal Processing, vol. 86, no. 3, pp. 549-571, 2006.

[32] H. Rauhut, "Circulant and Toeplitz matrices in compressed sensing," in Proceedings of the Signal Processing with Adaptive Sparse Structured Representations (SPARS '09), 2009.

[33] M. C. Angelini, F. Ricci-Tersenghi, and Y. Kabashima, "Compressed sensing with sparse, structured matrices," in Proceedings of the 50th Annual Allerton Conference on Communication, Control, and Computing (Allerton '12), pp. 808-814, 2012.

[34] C. Schüldt, I. Laptev, and B. Caputo, "Recognizing human actions: a local SVM approach," in Proceedings of the 17th International Conference on Pattern Recognition (ICPR '04), pp. 32-36, August 2004.

[35] S.-Z. Qin, H.-T. Su, and T. J. McAvoy, "Comparison of four neural net learning methods for dynamic system identification," IEEE Transactions on Neural Networks, vol. 3, no. 1, pp. 122-130, 1992.

[36] Y. Cai, T. Huang, L. Hu, X. Shi, L. Xie, and Y. Li, "Prediction of lysine ubiquitination with mRMR feature selection and analysis," Amino Acids, vol. 42, no. 4, pp. 1387-1395, 2012. 

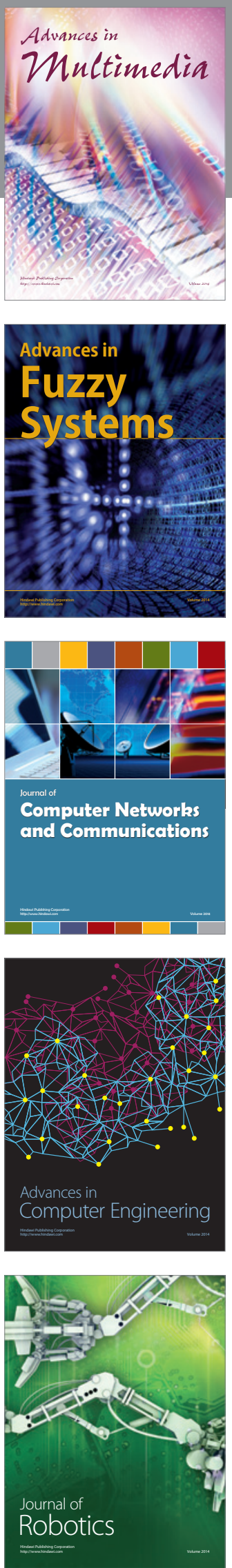

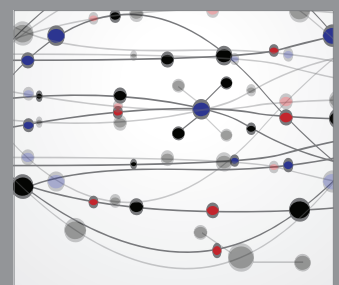

The Scientific World Journal
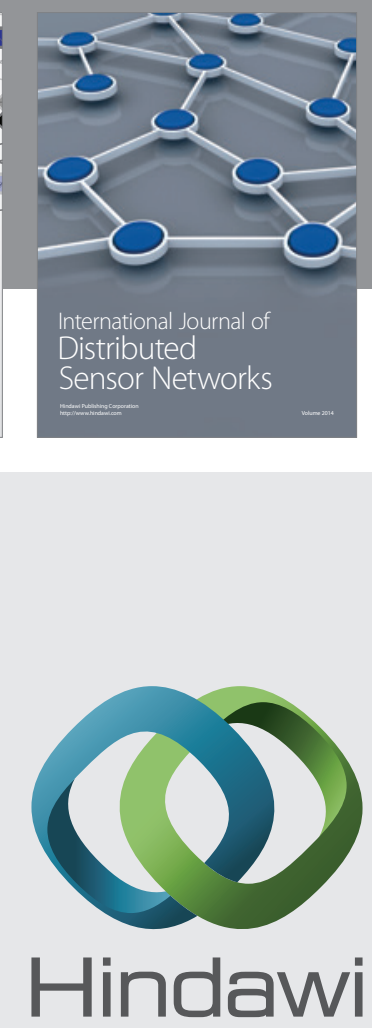

Submit your manuscripts at

http://www.hindawi.com
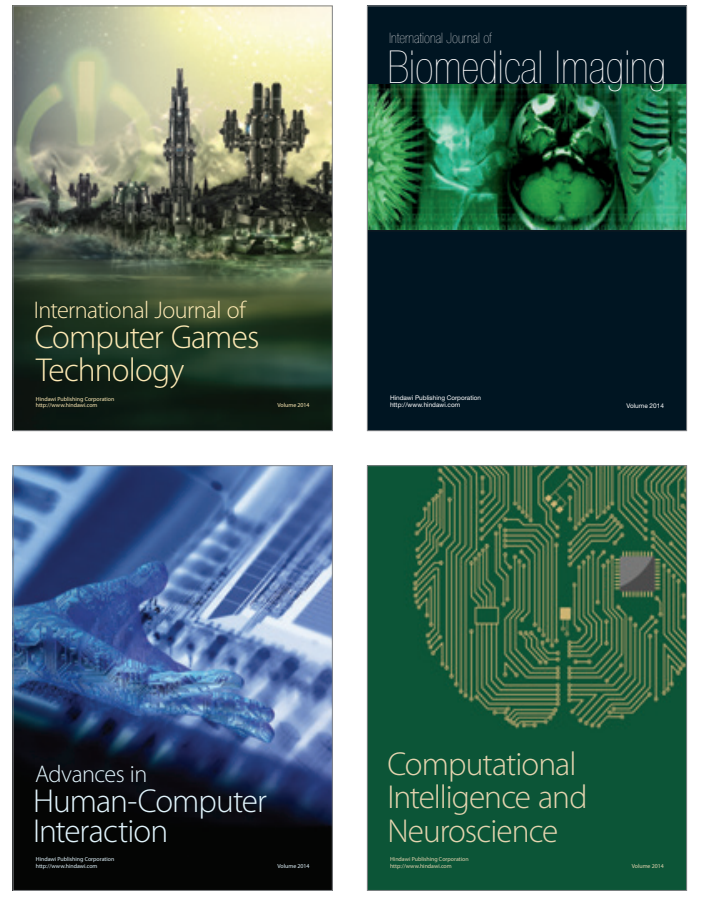
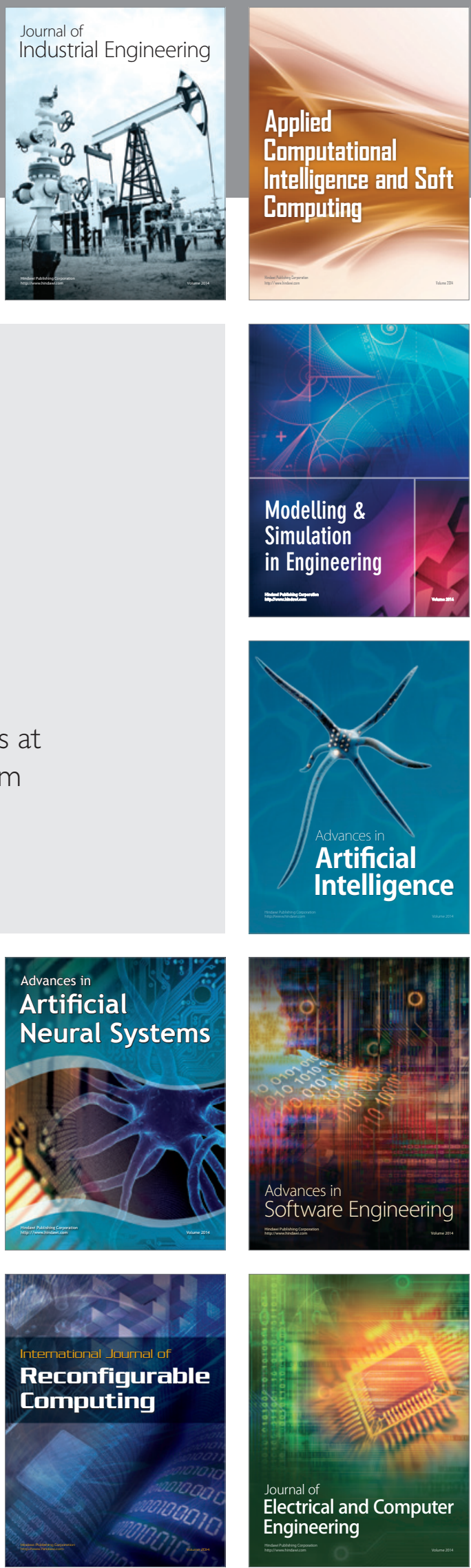